\title{
A Novel Blind Channel Estimation for a 2x2 MIMO System
}

\author{
Xia LIU ${ }^{1}$, Marek E. BIALKOWSKI ${ }^{2}$, Feng WANG ${ }^{1}$ \\ ${ }^{1}$ Student Member IEEE, School of ITEE, The University of Queensland, Brisbane, Australia \\ ${ }^{2}$ Fellow IEEE, School of ITEE, The University of Queensland, Brisbane, Australia \\ Email: \{xialiu,meb, fwang\}@itee.uq.edu.au \\ Received May 18, 2009; revised June 20, 2009; accepted July 17, 2009
}

\begin{abstract}
A novel blind channel estimation method based on a simple coding scheme for a 2 by 2 multiple input multiple output (MIMO) system is described. The proposed algorithm is easy to implement in comparison with conventional blind estimation algorithms, as it is able to recover the channel matrix without performing singular value decomposition (SVD) or eigenvalue decomposition (EVD). The block coding scheme accompanying the proposed estimation approach requires only a block encoder at the transmitter without the need of using the decoder at the receiver. The proposed block coding scheme offers the full coding rate and reduces the noise power to half of its original value. It eliminates the phase ambiguity using only one additional pilot sequence.
\end{abstract}

Keywords: MIMO, Channel Estimation, Semi-Blind Channel Estimation, Phase Ambiguity

\section{Introduction}

Multiple Input Multiple Output (MIMO) signal transmission schemes are attractive for high-speed data transmission in wireless communication systems because they offer an increased data throughput (capacity) without increasing operational bandwidth [1,2]. Also they are capable to enhance the quality of signal transmission through the use of transmitter or receiver diversity. These advantages are possible under the condition that the MIMO channel state information (CSI) is available at the receiver. Traditionally, CSI can be acquired by sending training sequences (also known as pilot signals) evenly spaced along a block of transmit symbols. The disadvantage of this approach is that the training sequences take up the precious bandwidth. In order to save the bandwidth and increase spectral efficiency, blind and semiblind channel estimation methods can be applied to obtain the CSI.

Several blind channel estimation methods have been described in $[3,4]$. These methods are based on the subspace algorithm [5], which utilizes the orthogonality between the channel matrix and the Sylvester matrixformed noise subspace. There are several drawbacks of subspace-based MIMO channel estimation methods. One is that they suffer from so-called multi-dimensional ambiguity. As a result, several pilot sequences are needed to eliminate this ambiguity. Two, in order to compensate for extra degrees of freedom in the noise subspace when the number of transmit antennas is smaller than the number of antennas at the receiver, the pre-coding is required $[3,4]$. Also, EVD is an inherent part of the algorithm, which leads to high implementation complexities.

In [6,7], a semi-blind channel estimation method employing orthogonal pilot maximum likelihood (OPML) estimator has been proposed. The method performs singular value decomposition (SVD) to the received signal correlation matrix to estimate the 'whitening' matrix of channel. By using the 'whitening' matrix, the OPML estimator shows a $1 \mathrm{~dB}$ improvement of bit error rate (BER) compared to the conventional least squares (LS) training scheme if the same length of training sequence is used. However, it still requires a large number of training symbols to achieve the same performance as LS. Furthermore, SVD has to be applied twice to obtain the 'whitening' matrix and the rotation matrix. These operations lead to the increased computational complexity.

The work in [8] presents a new SVD-based blind channel estimation scheme which uses a simple block pre-coding structure. The advantage of this approach is that CSI can be recovered without ambiguity if the 
proper modulation is applied. Another advantage of this scheme is that no block decoder is needed at the receiver. These advantages are gained at the expense of the coding rate. The coding rate decreases as more transmitting antennas are used. In particular, for a 2x2 MIMO system the code rate is $1 / 2$, which results in wasting of the precious spectrum.

In this paper, we propose a novel blind channel estimation algorithm, which is of much lesser complexity than those based on SVD or EVD. Its important feature is that it preserves the advantages of the coding scheme described in [8] without sacrificing the coding rate. The new scheme offers a full coding rate (coding rate is equal to 1) when the number of transmitting antennas is equal to the number of receiving antennas. In the case of a $2 \times 2$ MIMO system, this coding scheme reduces the noise power to the half of the original noise power. This scheme exhibits the phase ambiguity. However, it can be eliminated using only one extra pilot sequence.

The rest of the paper is organized as follows. In Section 2, a model of MIMO system employing a block coding scheme is introduced. A new blind channel estimation method is described in Section 3. The solution of eliminating the phase ambiguity is given in Section 4. Simulation results are presented in Section 5. Section 6 concludes the paper.

\section{System Description \& Coding Scheme}

In this paper, a narrow band block fading channel is assumed. The number of transmitting and receiving antennas is denoted as $N_{t}$ and $N_{r}$, respectively. Thus the channel $H$ is the $N_{r} \times N_{t}$ dimension channel matrix with $h_{i j}$ representing complex response between the $i$-th receiving antenna and the $j$-th transmitting antenna. In further considerations $N_{t}$ is assumed to be equal to $N_{r}$.

The input symbols at transmitter can be represented by

$$
X=\left\{x_{1}, x_{2}, x_{3}, \ldots . .\right\}
$$

where $X$ stands for independent identically distributed (i.i.d) Gaussian random signals with zero mean and the variance matrix given by $E\left\{x_{n} x_{m}{ }^{H}\right\}=\left\{\begin{array}{cc}\sigma_{s}^{2}, & n=m \\ 0, & n \neq m\end{array}\right.$, where $E\{\}$ implies the expectation and $\sigma_{\mathrm{s}}{ }^{2}$ is the power of one symbol.
The symbols are encoded using a block encoder structure before being transmitted. As a result, the $i$-th symbol block is an element of matrix group $A_{i} \in C^{N_{r} \times N_{r} N_{t}}=C^{2 \times 4}$.

The data received at the other end of the communication channel is affected by the channel properties and an additive noise. Therefore the relationship between the transmitted encoded symbols and received data is given as:

$$
Y_{i}=H A_{i}+N_{i}
$$

where $Y_{i}$ is the $N_{\mathrm{r}} \mathrm{x} N_{\mathrm{r}} N_{\mathrm{t}}$ received signal matrix and $N_{i}$ is the $N_{\mathrm{r}} \mathrm{x} N_{\mathrm{r}} N_{\mathrm{t}}$ (i.i.d) Gaussian random noise matrix with zero mean.

The coded output of the transmitter can be written as:

$$
A_{i}=\left[\begin{array}{llll}
A_{4 i+1} & A_{4 i+2} & A_{4 i+3} & A_{4 i+4}
\end{array}\right]
$$

where

$$
\begin{aligned}
& A_{4 i+1}=\operatorname{diag}\left(U_{1}\right) X_{2 i+1}^{T}, \\
& A_{4 i+2}=\operatorname{diag}\left(U_{2}\right) X_{2 i+1}^{T}, \\
& A_{4 i+4}=\operatorname{diag}\left(U_{2}\right) X_{2 i+2}^{T}, \quad i=0,1,2, \ldots \ldots, n
\end{aligned}
$$

and $X_{2 i+1}=\left[\begin{array}{ll}x_{4 i+1} & x_{4 i+2}\end{array}\right]$ and $X_{2 i+2}=\left[\begin{array}{ll}x_{4 i+3} & x_{4 i+4}\end{array}\right]$. $U=\left[\begin{array}{ll}U_{1}^{T} & U_{2}^{T}\end{array}\right], U_{1}=\left[\begin{array}{ll}1 & 1\end{array}\right], U_{2}=\left[\begin{array}{ll}1 & -1\end{array}\right]$ represent the encoder structure.

Therefore, the transmitted coded signals are

$$
\begin{aligned}
& A_{4 i+1}=\left[\begin{array}{l}
x_{4 i+1} \\
x_{4 i+2}
\end{array}\right], A_{4 i+2}=\left[\begin{array}{c}
x_{4 i+1} \\
-x_{4 i+2}
\end{array}\right] \\
& A_{4 i+3}=\left[\begin{array}{l}
x_{4 i+3} \\
x_{4 i+4}
\end{array}\right], A_{4 i+4}=\left[\begin{array}{c}
x_{4 i+3} \\
-x_{4 i+4}
\end{array}\right]
\end{aligned}
$$

or

$$
A_{i}=\left[\begin{array}{cccc}
x_{4 i+1} & x_{4 i+1} & x_{4 i+3} & x_{4 i+3} \\
x_{4 i+2} & -x_{4 i+2} & x_{4 i+4} & -x_{4 i+4}
\end{array}\right]
$$

From expression (6), one can observe that 4 symbols are sent in 4 symbol periods during one block. Therefore, the code rate is 1 .

The received signal blocks can be written as (7):

$$
\begin{aligned}
Y_{i} & =\left[\begin{array}{llll}
y_{11}^{2 i+1} & y_{12}^{2 i+1} & y_{11}^{2 i+2} & y_{12}^{2 i+2} \\
y_{21}^{2 i+1} & y_{22}^{2 i+1} & y_{21}^{2 i+2} & y_{22}^{2 i+2}
\end{array}\right] \\
& =\left[\begin{array}{llll}
h_{11} x_{4 i+1}+h_{12} x_{4 i+2} & h_{11} x_{4 i+1}-h_{12} x_{4 i+2} & h_{11} x_{4 i+3}+h_{12} x_{4 i+4} & h_{11} x_{4 i+3}-h_{12} x_{4 i+4} \\
h_{21} x_{4 i+1}+h_{22} x_{4 i+2} & h_{21} x_{4 i+1}-h_{22} x_{4 i+2} & h_{21} x_{4 i+3}+h_{22} x_{4 i+4} & h_{21} x_{4 i+3}-h_{22} x_{4 i+4}
\end{array}\right]+N_{i} \\
& =H A_{i}+N_{i}
\end{aligned}
$$


in which $\quad N_{i}=\left[\begin{array}{llll}n_{11}^{2 i+1} & n_{12}^{2 i+1} & n_{11}^{2 i+2} & n_{12}^{2 i+2} \\ n_{21}^{2 i+1} & n_{22}^{2 i+1} & n_{21}^{2 i+2} & n_{22}^{2 i+2}\end{array}\right]$ is the

random Gaussian noise matrix. An equivalent representation of (7) is given by (8):

$$
\begin{aligned}
& y_{11}^{2 i+1}=h_{11} x_{4 i+1}+h_{12} x_{4 i+2}+n_{11}^{2 i+1} \\
& y_{12}^{2 i+1}=h_{11} x_{4 i+1}-h_{12} x_{4 i+2}+n_{12}^{2 i+1} \text { and } \begin{array}{l}
y_{21}^{2 i+1}=h_{21} x_{4 i+1}+h_{22} x_{4 i+2}+n_{21}^{2 i+1} \\
y_{22}^{2 i+1}=h_{21} x_{4 i+1}-h_{22} x_{4 i+2}+n_{22}^{2 i+1}
\end{array} \\
& y_{11}^{2 i+2}=h_{11} x_{4 i+3}+h_{12} x_{4 i+4}+n_{11}^{2 i+2} \\
& y_{12}^{2 i+2}=h_{11} x_{4 i+3}-h_{12} x_{4 i+4}+n_{12}^{2 i+2} \text { and } \begin{array}{l}
y_{21}^{2 i+2}=h_{21} x_{4 i+3}+h_{22} x_{4 i+4}+n_{21}^{2 i+2} \\
y_{22}^{2 i+2}=h_{21} x_{4 i+3}-h_{22} x_{4 i+4}+n_{22}^{2 i+2}
\end{array}
\end{aligned}
$$

By linking (8) directly to individual channel matrix elements, one obtains:

$$
\begin{aligned}
& h_{11} x_{4 i+1}=\frac{y_{11}^{2 i+1}+y_{12}^{2 i+1}}{2}-\frac{n_{12}^{2 i+1}+n_{11}^{2 i+1}}{2}, \\
& h_{12} x_{4 i+2}=-\frac{y_{12}^{2 i+1}-y_{11}^{2 i+1}}{2}+\frac{n_{12}^{2 i+1}-n_{11}^{2 i+1}}{2} \\
& h_{21} x_{4 i+1}=\frac{y_{21}^{2 i+1}+y_{22}^{2 i+1}}{2}-\frac{n_{22}^{2 i+1}+n_{21}^{2 i+1}}{2}, \\
& h_{22} x_{4 i+2}=-\frac{y_{22}^{2 i+1}-y_{21}^{2 i+1}}{2}+\frac{n_{22}^{2 i+1}+n_{21}^{2 i+1}}{2} \\
& h_{11} x_{4 i+3}=\frac{y_{11}^{2 i+2}+y_{12}^{2 i+2}}{2}-\frac{n_{12}^{2 i+2}+n_{11}^{2 i+2}}{2}, \\
& h_{12} x_{4 i+4}=-\frac{y_{12}^{2 i+2}-y_{11}^{2 i+2}}{2}+\frac{n_{12}^{2 i+2}+n_{11}^{2 i+2}}{2} \\
& h_{21} x_{4 i+3}=\frac{y_{21}^{2 i+2}+y_{22}^{2 i+2}}{2}-\frac{n_{22}^{2 i+2}+n_{21}^{2 i+2}}{2} \\
& h_{22} x_{4 i+4}=-\frac{y_{22}^{2 i+2}-y_{21}^{2 i+2}}{2}+\frac{n_{22}^{2 i+2}-n_{21}^{2 i+2}}{2}
\end{aligned}
$$

As a result, the relationship between the raw (transmitted) data and the received data is given by (13):

$$
\bar{Y}_{i}=\bar{H} \bar{X}_{i}+\bar{N}_{i}
$$

in which the individual terms are identified by (14)

$$
\left[\begin{array}{l}
\frac{y_{11}^{2 i+1}+y_{12}^{2 i+1}}{2} \\
\frac{y_{11}^{2 i+1}-y_{12}^{2 i+1}}{2} \\
\frac{y_{11}^{2 i+2}+y_{22}^{2 i+2}}{2} \\
\frac{y_{21}^{2 i+2}-y_{22}^{2 i+2}}{2}
\end{array}\right]=\left[\begin{array}{cccc}
h_{11} & 0 & 0 & 0 \\
0 & h_{12} & 0 & 0 \\
0 & 0 & h_{21} & 0 \\
0 & 0 & 0 & h_{22}
\end{array}\right]\left[\begin{array}{l}
x_{4 i+1} \\
x_{4 i+2} \\
x_{4 i+3} \\
x_{4 i+4}
\end{array}\right]+\left[\begin{array}{c}
n_{4 i+1} \\
n_{4 i+2} \\
n_{4 i+3} \\
n_{4 i+4}
\end{array}\right]
$$

where

$$
\begin{aligned}
& n_{4 i+1}=\frac{n_{12}^{2 i+1}+n_{11}^{2 i+1}}{2}, n_{4 i+2}=\frac{n_{11}^{2 i+1}-n_{12}^{2 i+1}}{2}, \\
& n_{4 i+3}=\frac{n_{22}^{2 i+2}+n_{21}^{2 i+2}}{2}, n_{4 i+4}=\frac{n_{21}^{2 i+2}-n_{22}^{2 i+2}}{2}
\end{aligned}
$$

Due to the fact that the elements in $N i$ represent a Gaussian random noise also the elements in $\overline{N_{i}}$ obey the Gaussian distribution. However, the average power of each element in $\bar{N}_{i}$ is half of that in $N_{i}$. In this case, the noise power is suppressed by the coding scheme.

\section{Blind Channel Estimation}

The blind channel estimation requires the knowledge of the correlation matrix of $\bar{Y}$, which is given as:

$$
R_{i}=E\left\{\bar{Y}_{i} \bar{Y}_{i}^{H}\right\}=\bar{H} E\left\{\bar{X}_{i} \bar{X}_{i}^{H}\right\} \bar{H}^{H}+E\left\{\bar{N}_{i} \bar{N}_{i}^{H}\right\}
$$

Because the power of each element in $\overline{N_{i}}$ is half of that in the noise matrix $N_{i}$, then Equation (16) can be rewritten as:

$$
R_{i}=E\left\{\bar{Y}_{i} \bar{Y}_{i}^{H}\right\}=\bar{H} E\left\{\bar{X}_{i} \bar{X}_{i}^{H}\right\} \bar{H}^{H}+\frac{1}{2} E\left\{N_{i} N_{i}^{H}\right\}
$$

If the information symbol sequence is of unit power then (17) becomes:

$$
R_{i}=\bar{H} \overline{\overline{X_{i}}} \bar{H}^{H}+\frac{1}{2} E\left\{N_{i} N_{i}{ }^{H}\right\}
$$

where

$$
\overline{\bar{X}}=E\left\{\bar{X}_{i} \bar{X}_{i}^{H}\right\}=I_{N_{r} N_{t} \times N_{r} N_{t}}
$$

Thus (18) can be converted to (19)

$$
R_{i}=\bar{H} \bar{H}^{H}+\frac{1}{2} E\left\{N_{i} N_{i}^{H}\right\}
$$

By introducing $\bar{h}=\operatorname{vec}(H)$, the following holds: 


$$
\bar{H} \bar{H}^{H}=\operatorname{diag}\left(|\bar{h}|^{2}\right)
$$

where vec (.) means vector operation in which columns of $H$ are stacked on top of each other and |.| denotes the absolute value.

$$
|\bar{h}|^{2}=\left[\begin{array}{lll}
\left|h_{11}\right|^{2} & \left|h_{12}\right|^{2} \quad \cdots \quad\left|h_{N_{r} N_{t}}\right|^{2}
\end{array}\right]
$$

As a result

$$
R_{i}=\operatorname{diag}\left(|\bar{h}|^{2}\right)+\frac{1}{2} E\left\{N_{i} N_{i}^{H}\right\}
$$

The estimation of $\bar{h}$ is equivalent to finding the roots of the diagonal elements in $R$.

To obtain the solution, the square-root algorithm can be applied. One problem that is faced using this approach is that it introduces the phase ambiguity in the estimated $\bar{h}$. This is because the square roots are obtained for the norms of the elements of the channel matrix $H$. In the next section, a method for the phase ambiguity elimination is described.

\section{Phase Ambiguity Elimination}

It is apparent that the proposed blind channel estimation algorithm provides the information about the estimated norm of each element in channel matrix $H$ of the $2 \times 2$ MIMO system as shown by the following.

$$
\hat{H}=\left[\begin{array}{ll}
\left|\hat{h}_{11}\right| & \left|\hat{h}_{12}\right| \\
\left|\hat{h}_{21}\right| & \left|\hat{h}_{22}\right|
\end{array}\right]
$$

Now the task is to obtain the phases of these elements. By sending one pilot sequence $P$ where

$$
P=\left[\begin{array}{l}
P_{1} \\
P_{2}
\end{array}\right]=\left[\begin{array}{l}
1 \\
1
\end{array}\right]
$$

one obtains

$$
Y_{p}=H P+N
$$

in which $Y_{p}$ and $P$ are known. More specifically, we have

$$
\begin{aligned}
Y_{p} & =\left[\begin{array}{l}
X_{p}^{1}+j \cdot Y_{p}^{1} \\
X_{p}^{2}+j \cdot Y_{p}^{2}
\end{array}\right] \\
& =\left[\begin{array}{ll}
\left|h_{11}\right| e^{j \theta_{11}} & \left|h_{12}\right| e^{j \theta_{12}} \\
\left|h_{21}\right| e^{j \theta_{21}} & \left|h_{22}\right| e^{j \theta_{22}}
\end{array}\right]\left[\begin{array}{l}
1 \\
1
\end{array}\right]+\left[\begin{array}{l}
N_{1} \\
N_{2}
\end{array}\right]
\end{aligned}
$$

By replacing $\left|h_{N_{r} N_{t}}\right|$ in (25) by the estimated values, the following is obtained

$$
\begin{aligned}
X_{p}^{1} & =\left|\hat{h}_{11}\right| \cos \theta_{11}+\left|\hat{h}_{12}\right| \cos \theta_{12} \\
Y_{p}^{1} & =\left|\hat{h}_{11}\right| \sin \theta_{11}+\left|\hat{h}_{12}\right| \sin \theta_{12} \\
X_{p}^{2} & =\left|\hat{h}_{21}\right| \cos \theta_{21}+\left|\hat{h}_{22}\right| \cos \theta_{22} \\
Y_{p}^{2} & =\left|\hat{h}_{21}\right| \sin \theta_{21}+\left|\hat{h}_{22}\right| \sin \theta_{22}
\end{aligned}
$$

To determine $\theta_{11}, \theta_{12}, \theta_{21}$ and $\theta_{22}$, Equations (9)(10) and (11)(12) are used in which (9)/(10) and (11)/(12) are formed. This operation results in the following

$$
\begin{gathered}
\frac{h_{11}}{h_{21}}=\frac{\left|h_{11}\right|}{\left|h_{21}\right|} e^{j\left(\theta_{11}-\theta_{21}\right)}=\frac{\left|h_{11}\right|}{\left|h_{21}\right|} e^{i \sigma_{1}}=\frac{\frac{y_{11}^{2 i+1}+y_{12}^{2 i+1}}{2}-\frac{n_{12}^{2 i+1}+n_{11}^{2 i+1}}{2}}{\frac{y_{21}^{2 i+1}+y_{22}^{2 i+1}}{2}-\frac{n_{22}^{2 i+1}+n_{21}^{2 i+1}}{2}}, \\
\frac{h_{12}}{h_{22}}=\frac{\left|h_{12}\right|}{\left|h_{22}\right|} e^{j\left(\theta_{12}-\theta_{22}\right)}=\frac{\left|h_{12}\right|}{\left|h_{22}\right|} e^{j \sigma_{2}}=\frac{\frac{y_{12}^{2 i+1}-y_{11}^{2 i+1}}{2}-\frac{n_{12}^{2 i+1}+n_{11}^{2 i+1}}{2}}{\frac{y_{22}^{2 i+1}-y_{21}^{2 i+1}}{2}-\frac{n_{22}^{2 i+1}+n_{21}^{2 i+1}}{2}} \\
\frac{h_{11}}{h_{21}}=\frac{\left|h_{11}\right|}{\left|h_{21}\right|} e^{j\left(\theta_{1}-\theta_{21}\right)}=\frac{\left|h_{11}\right|}{\left|h_{21}\right|} e^{j \sigma_{1}}=\frac{\frac{y_{11}^{2 i+2}+y_{12}^{2 i+2}}{2}-\frac{n_{12}^{2 i+2}+n_{11}^{2 i+2}}{2}}{\frac{y_{21}^{2 i+2}+y_{22}^{2 i+2}}{2}-\frac{n_{22}^{2 i+2}+n_{21}^{2 i+2}}{2}}, \\
\frac{h_{12}}{h_{22}}=\frac{\left|h_{12}\right|}{\left|h_{22}\right|} e^{j\left(\theta_{2}-\theta_{22}\right)}=\frac{\left|h_{12}\right|}{\left|h_{22}\right|} e^{j \sigma_{2}}=\frac{\frac{y_{12}^{2 i+2}-y_{11}^{2 i+2}}{2}-\frac{n_{12}^{2 i+2}+n_{11}^{2 i+2}}{2}}{\frac{y_{22}^{2 i+2}-y_{21}^{2 i+2}}{2}-\frac{n_{22}^{2 i+2}+n_{21}^{2 i+2}}{2}}
\end{gathered}
$$


where $\sigma_{1}=\theta_{11}-\theta_{21}$ and $\sigma_{2}=\theta_{12}-\theta_{22}$.

Using Equations (32) and (33), $\sigma_{1}$ and $\sigma_{2}$ can be estimated by including noise impact. Then $\theta_{11}, \theta_{21}$ and $\theta_{12}$, $\theta_{22}$ can be expressed using the estimated $\sigma_{1}$ and $\sigma_{2}$ as,

$$
\begin{aligned}
& \theta_{21}=\hat{\sigma}_{1}-\theta_{11} \\
& \theta_{22}=\hat{\sigma}_{2}-\theta_{12}
\end{aligned}
$$

By substituting (34) into Equations (32) and (35) into Equations (33), the phases $\theta_{11}, \theta_{21}, \theta_{12}$ and $\theta_{22}$ can be determined. Therefore, the phase ambiguity can be resolved.

\section{Simulation Results}

The validity of the proposed blind channel estimation algorithm for a $2 \times 2$ MIMO is investigated via computer simulations. For reference purposes, comparisons are made with a training-based channel estimation using the least square (LS) method and a semi-blind channel estimation using an orthogonal pilot maximum likelihood (OPML) algorithm. The following is the necessary information that is used to perform comparisons with the alternative channel estimation algorithms.

The performance of the proposed channel estimation is assessed in terms of mean square error (MSE) as given by

$$
M S E=E\left\{\|H-\hat{H}\|_{F}^{2}\right\}
$$

in which $\|.\|_{F}^{2}$ stands for the Frobenius norm.

In the LS method, the estimated channel matrix is given as [10],

$$
\hat{H}_{L S}=Y P^{\dagger}
$$

where $\{.\}^{\dagger}$ stands for the pseudo-inverse operation.

The MSE of LS method is given as

$$
M S E_{L S}=E\left\{\left\|H-\hat{H}_{L S}\right\|_{F}^{2}\right\}
$$

According to [11] and [12], the minimum value of MSE for the LS method is given as

$$
M S E_{\min }^{L S}=\frac{M_{t}^{2} M_{r}}{\rho}
$$

in which $\rho$ stands for transmitted power to noise ratio in the training mode. Here we assume that the SNR in the proposed estimation scenario is equal to $\rho$.

In [6] and [7], a WR-based semi-blind channel estimation method was introduced. Following that method, the MIMO channel matrix $H$ can be decomposed by applying the singular value (SV) decomposition

$$
H \stackrel{S V D}{\longrightarrow} P \Sigma Q^{H}
$$

where $P$ and $Q$ are two singular vectors corresponding to eigenvalues. $\Sigma$ represents eigenvalues of $H$ by the diagonal matrix. For both $P$ and $Q$ the following properties hold: $P P^{H}=P^{H} P=I$ and $Q Q^{H}=Q^{H} Q=I$. The whitening matrix $W$ is given by $W=P \Sigma$ and $Q$ is the rotation matrix. The whitening matrix $W$ can be obtained blindly by computing the second-order statistics of a received signal. Details are given in Section 2.3 of [13] and thus are not repeated here. The matrix $W$ is assumed to be perfectly known at the receiver. Training sequences are used for estimating the unitary matrix $Q$. It has already been proved in [6] and [7] that such a unitary matrix $Q$ helps increasing estimation gains because it uses a smaller number of parameters.

To estimate the rotation matrix $Q$, several algorithms can be applied. The orthogonal pilot maximum likelihood (OPML) algorithm offers the best performance, as demonstrated in [6]. In this algorithm, the training matrix $X_{p}$ is set to have orthogonal properties with unit power and length equal to $N_{t}, X_{p}^{H} X_{p}=X_{p} X_{p}^{H}=I$. The OPML estimator is expressed as (36),

$$
\begin{aligned}
& \arg \min \left\|Y_{P}-W Q^{H} X_{P}\right\|^{2} \\
& \text { subj. to } Q Q^{H}=I
\end{aligned}
$$

where $Q$ is obtained by minimizing the likelihood.

Let $\hat{M}=W^{H} Y_{p} X_{p}^{H}$, then by applying SVD to $\hat{M}$, we have

$$
\hat{\mathrm{U}}_{\mathrm{M}} \hat{\Sigma}_{M} \hat{V}_{M}^{H}=S V D(\hat{M})
$$

It can be shown that the estimated $\hat{Q}$ of $Q$ that minimizes the likelihood is given as [6]:

$$
\hat{Q}=\hat{V}_{M} \hat{U}_{M}^{H}
$$

The channel matrix $H$ is then estimated as

$$
\hat{H}=W \hat{Q}^{H}
$$

In Figure 1, the performance of LS method, OPML algorithm and the proposed blind estimation method for a $2 \times 2$ MIMO system are presented. The length of training sequences used by LS method and OPML algorithm is set to 2 .

From Figure 1, one can see that when the number of received symbols increases, the blind estimation accuracy is improved. The performance of blind channel estimation is always better than offered by LS. This is mainly because the proposed algorithm reduces the noise power to half of the original one. When making comparison with OPML algorithm, one finds that the per- 


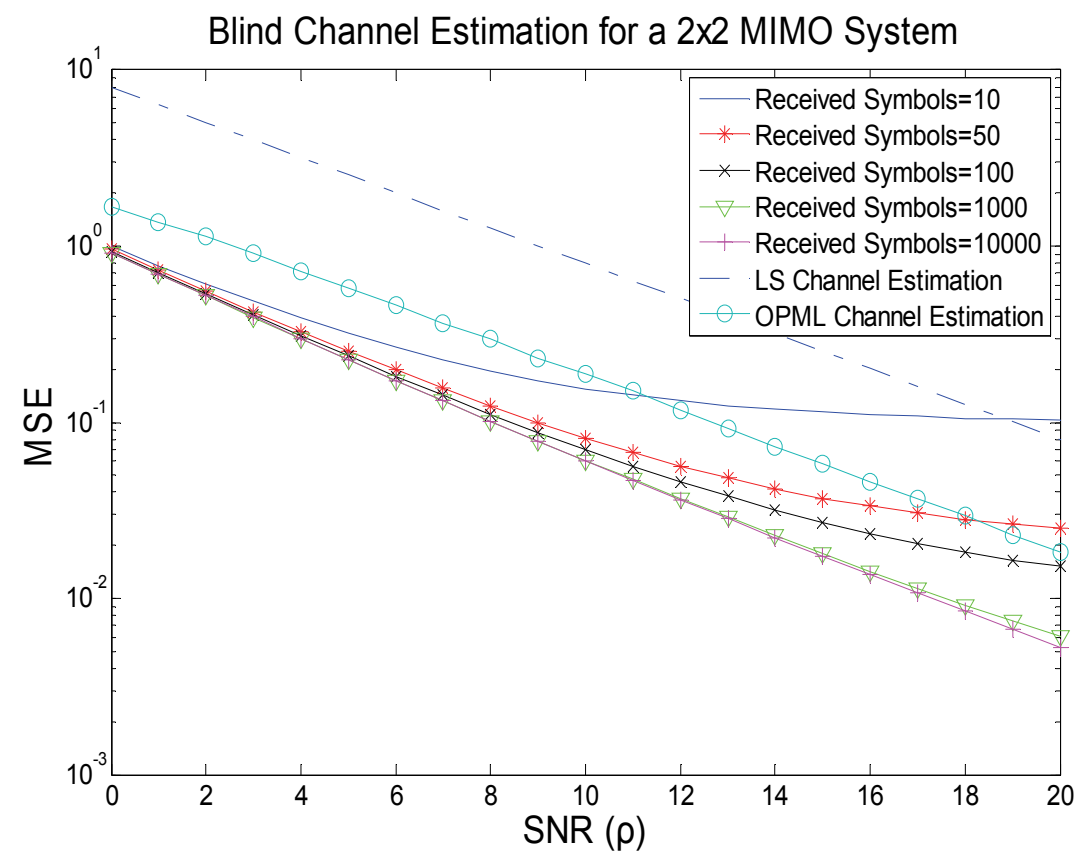

Figure 1. Performance of LS, OPML and newly proposed blind estimation algorithm for a $2 \times 2$ MIMO system for different values of SNR.

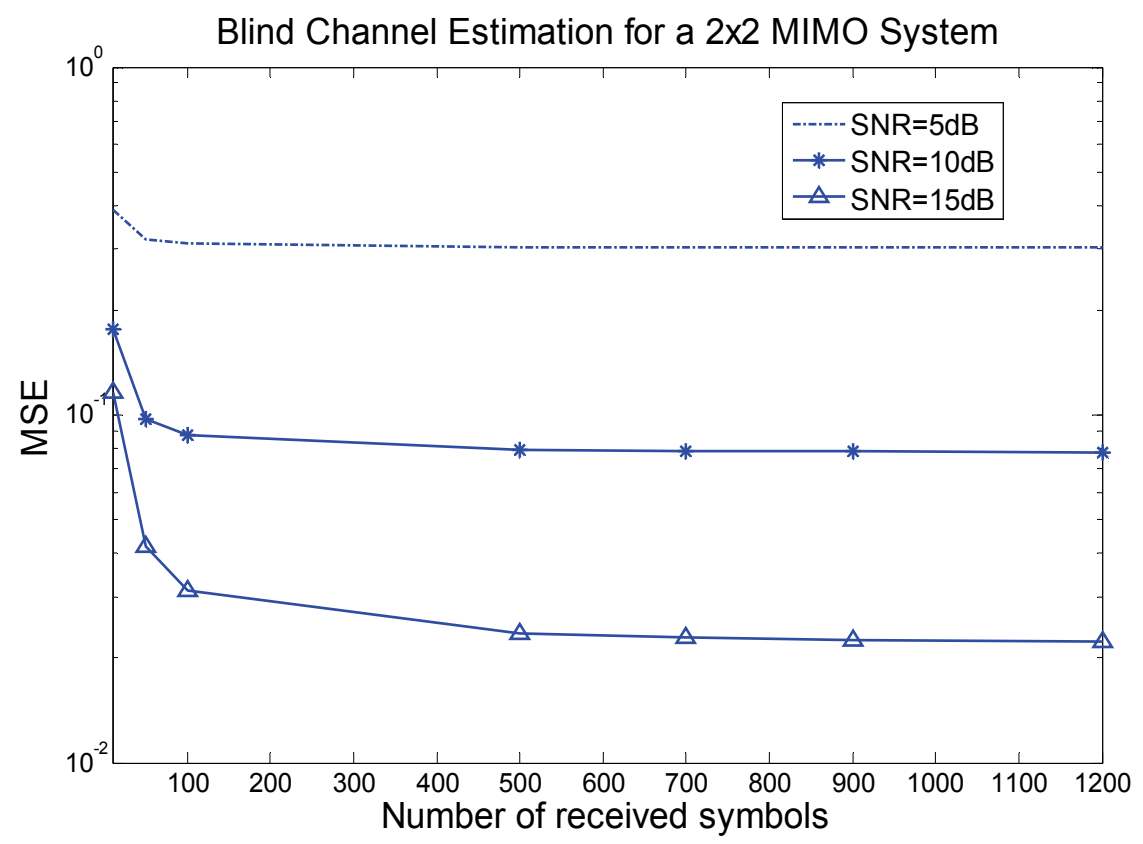

Figure 2. MSE as a function of number of received symbols for a newly proposed blind channel estimation method for a $2 \times 2$ MIMO system for different values of SNR.

formance of the proposed estimation algorithm is always better when the number of received symbols is equal or more than 100 .

Figure 2 illustrates the convergence of the blind channel estimation for a $2 \times 2$ MIMO system. It can be seen that for each of the three assumed values of SNR, the convergence occurs within the first 100 received symbols. The use of a larger number of symbols (100 to 500) provides only a slight improvement. The full convergence occurs at about 700 symbols. One can see that with 100 
received symbols the estimation is already very accurate irrespectively of the assumed value of SNR.

\section{Conclusions}

In this paper we have presented a novel blind algorithm for estimating a channel of a $2 \times 2$ MIMO. The proposed algorithm operates in conjunction with a suitable coding scheme and eliminates phase ambiguity for the estimated channel matrix coefficients. The coding scheme exhibits high spectral efficiency and reduces the noise power to the half of the original one that is present in a $2 \times 2 \mathrm{MIMO}$ system. The proposed algorithm involves the square-root operation which shows a low level of processing complexity. The simulation results prove a fast convergence rate for estimating the channel. The performance of the proposed algorithm is better than of the training-based Least Squares (LS) algorithm. Also it shows superiority over the Orthogonal Pilot Maximum Likelihood (OPML) semi-blind estimation algorithm when the number of received signal symbols exceeds 100 .

\section{References}

[1] Telatar, "Capacity of multiple antenna Gaussian channels," European Transactions on Telecommunications, Vol. 10, No. 6, pp. 585-595, November/December 1999.

[2] G. J. Foschini and M. J. Gans, "On limits of wireless communications in a fading environment when using multiple antennas," Wireless Personal Communications, Vol. 6, pp. 311-335, 1998.

[3] S. Zhou, B. Muquet, and G. B. Giannakis, "Subspace-based (semi-) blind channel estimation for block precoded space-time OFDM," IEEE Transactions on Signal Processing, Vol. 50, No. 5, pp. 1215-1228, May 2002.
[4] R. Zhang, "Blind OFDM channel estimation through linear Precoding: A subspace approach," in Proceedings Asilomar'02, Pacific Grove, CA, November 2002.

[5] E. Moulines, P. Duhamel, J. F. Cardoso, and S. Mayrargue, "Subspace methods for the blind identification of multichannel FIR filters," IEEE Transactions on Signal Processing, Vol. 43, pp. 516-525, February 1995.

[6] A. K. Jagannatham and B. D. Rao, "Whitening-rotationbased semi-blind MIMO channel estimation," IEEE Transactions on Signal Processing, Vol. 54, No. 3, March 2006.

[7] A. Jagannatham and B. D. Rao, "Constrained ML algorithms for semi-blind MIMO channel estimation," Proceedings of IEEE Communication Society Globecom, 2004.

[8] X. Liu and M. E. Bialkowski, "SVD-Based blind channel estimation for a MIMO OFDM system employing a simple block pre-coding scheme," Proceedings of IEEE Eurocon, Poland, 2007.

[9] S. Shahbazpanahi, A. B. Gershman, and J. H. Manton, "Closed-form blind MIMO channel estimation for orthogonal space-time block codes," IEEE Transactions on Signal Processing, Vol. 53, No. 12, December 2005.

[10] S. M. Kay, "Fundamentals of statistic signal processing: Estimation theory," Prentice-Hall, Incorporation, 1993.

[11] M. Biguesh and A. B. Gershman, "MIMO channel estimation: Optimal training and tradeoffs between estimation techniques," Proceedings ICC'04, Paris, France, June 2004.

[12] M. Biguesh and A. B. Gershman, "Training-based MIMO channel estimation: A study of estimator tradeoffs and optimal training signals," IEEE Transactions on Signal Processing, Vol. 54, No. 3, March 2000.

[13] A. K. Jagannatham and B. D. Rao, "A semi-blind technique for MIMO channel matrix estimation," Proceedings IEEE Workshop on SPAWC, Roma, Italy, 2003. 